\title{
High energy density physics at the Atomic Weapons Establishment
}

\author{
Andrew Randewich and Colin Danson \\ AWE, Aldermaston, Reading RG7 4PR, UK \\ (Received 3 October 2014; accepted 11 November 2014)
}

\begin{abstract}
The Atomic Weapons Establishment (AWE) is tasked with supporting Continuous At Sea Deterrence (CASD) by certifying the performance and safety of the national deterrent in the Comprehensive Test Ban Treaty (CTBT) era. This means that recourse to further underground testing is not possible, and certification must be achieved by supplementing the historical data with the use of computer calculation. In order to facilitate this, AWE operates some of the largest supercomputers in the UK. To validate the computer codes, and indeed the designers who are using them, it is necessary to carry out further experiments in the right regimes. An excellent way to meet many of the requirements for material property data and to provide confidence in the validity of the algorithms is through experiments carried out on high power laser facilities.
\end{abstract}

Keywords: high energy density physics; HEDP; high performance computing; HPC; high power lasers

\section{High performance computing}

Certification of the national deterrent in the Comprehensive Test Ban Treaty (CTBT) era must be achieved through the use of computer calculation ${ }^{[1]}$. The large supercomputers used at Atomic Weapons Establishment (AWE) are supplemented by high resilience data storage, high speed networks, visualization clusters and high end desktops that enable the supercomputers to be exploited effectively. The current systems have recently been upgraded to provide around two and a half petaflop/s (2.5 million billion floating point operations per second), and AWE has also recently procured several advanced architecture platforms in order to ensure that the most efficient (in terms of both cost and energy) hardware possible can be deployed in future years. Figure 1 shows AWE's high performance computer 'Blackthorn'.

This high performance computing (HPC) environment is of course useless without computer programmes or 'codes' to run on it. Multi-physics algorithms are therefore developed to solve the equations of compressible fluid flow coupled to transport algorithms and other required physics such as fusion burn. These state-of-the-art algorithms are in turn worthless without material property data. Perhaps unsurprisingly, it is particularly challenging to obtain the required material properties such as opacity, strength and equation of state in the extreme conditions pertinent to

Correspondence to: A. Randewich, AWE, Aldermaston, Reading RG7 4PR, UK. Email: Andrew.randewich@awe.co.uk nuclear warheads. The requirements span time scales of many orders of magnitude and pressures up to gigabars, with temperatures ranging up to thousands of electron volts $(\mathrm{keV}$, or tens of millions of degrees).

The regimes of hot dense matter (HDM) and warm dense matter (WDM) are pertinent, representing material that is heated while remaining at a density close to solid; similar conditions exist in the cores of large planets and in the Sun, see Figure 2. In some cases it is possible to harness the power of HPC to calculate the material properties in question, although this is non-trivial; the conditions of WDM sit between well understood regimes, in particular because the thermal energy of WDM ions is typically comparable with their potential energy. The physics is therefore intermediate to solid-state physics (dominated by interatomic potentials) and traditional plasma physics (dominated by thermal energy), so simplifying assumptions required for modelling will not be valid across all the required parameter space. In any case, it is always important to underpin calculated properties with measurements in appropriate conditions, and especially so in such a difficult regime. Once the material property data have been incorporated into the multi-physics codes, meaningful calculations become possible.

\section{High power lasers for high energy density physics}

High power lasers have been used at AWE for the study of high energy density conditions for almost forty years ${ }^{[2]}$. 


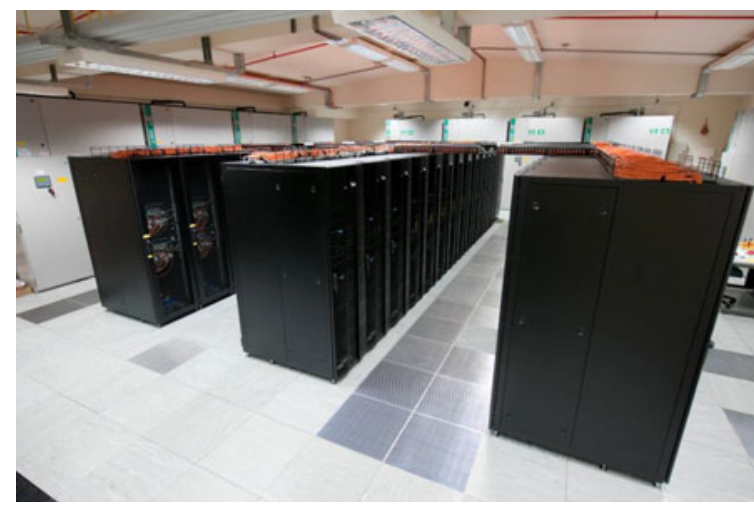

Figure 1. AWE's high performance computer 'Blackthorn'.

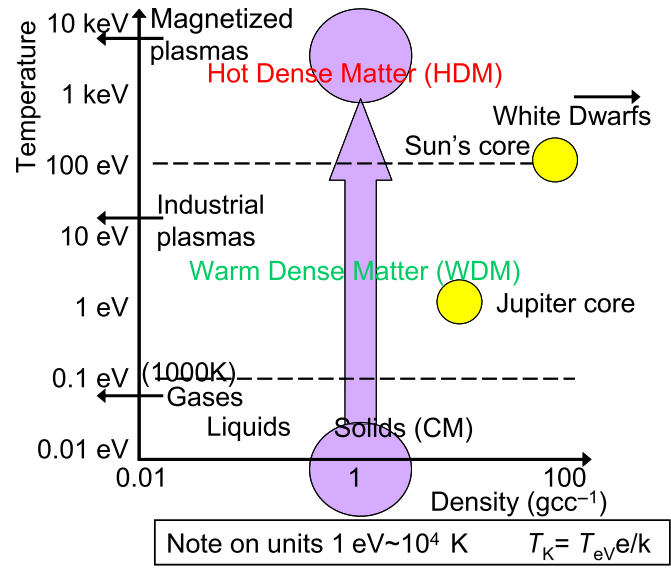

Figure 2. WDM and HDM are generated in planetary and solar cores as well as within nuclear weapons.

Following the laser fusion concept of Nuckolls ${ }^{[3]}$, AWE began modelling laser plasmas in the early 1970s. The concept of using a hohlraum of high atomic number material to convert laser energy to thermal x-rays rather than directly driving a capsule was developed and within ten years the HELEN laser ${ }^{[4]}$ was opened, see Figure 3. HELEN was initially a two-beam system, each delivering a terawatt of power (a kilojoule in a nanosecond). In parallel with HELEN experiments, the much higher energy drive available from underground nuclear tests was used as a platform to drive fusion experiments. While these experiments were successful in demonstrating the validity of the key science of laser fusion, this research was effectively terminated when nuclear testing ceased in 1991. Experiments in HELEN's target chamber (see Figure 4) continued, and the laser was upgraded at regular intervals, with the two beams being converted to green light, and a third beamline being added as a backlighter for diagnostic purposes.

By the year 2000 it was realized that HELEN had almost exhausted the phase space that it could access, and yet requirements remained for further data on material properties in hot dense plasmas and experiments to validate

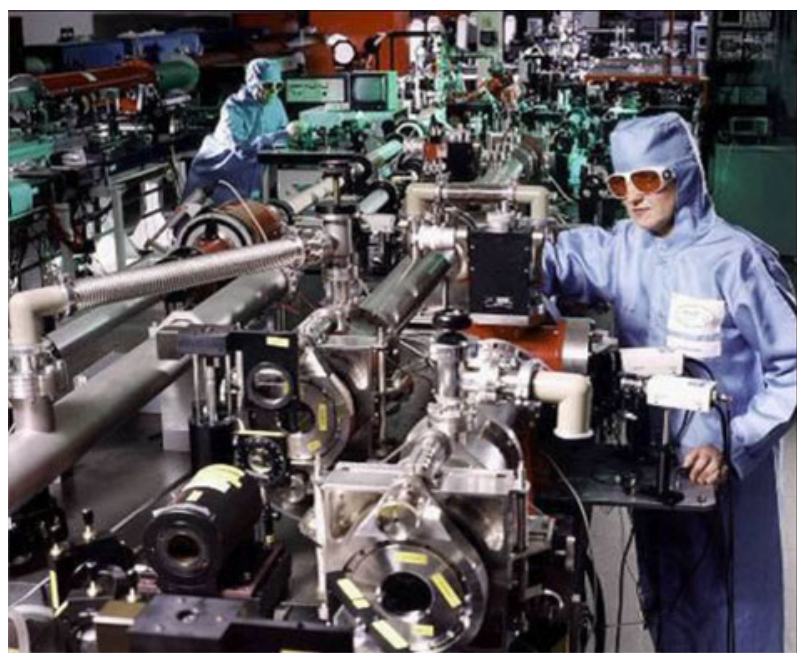

Figure 3. The HELEN laser was decommissioned in 2009 after demonstrating the feasibility of carrying out relevant high energy density physics (HEDP) experiments on laser systems.

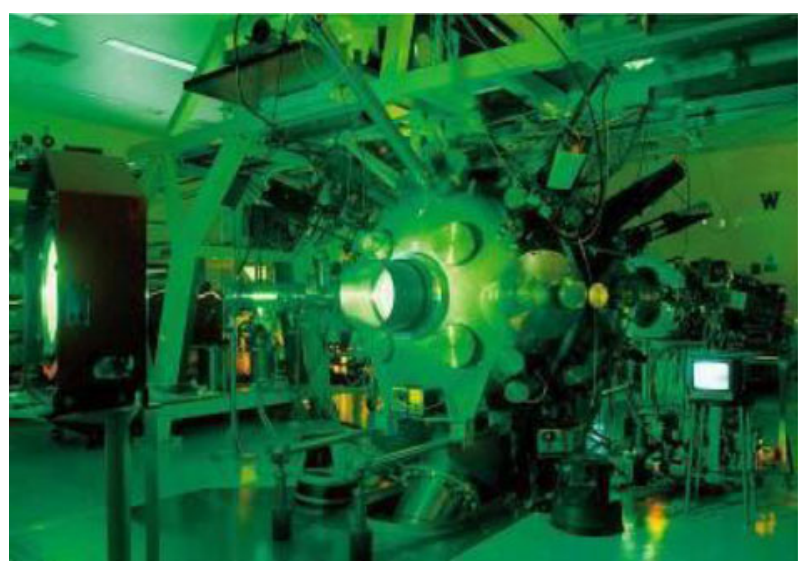

Figure 4. The HELEN target chamber.

calculations. The construction of a laser system with sufficient energy to compress and heat to the conditions of interest using nanosecond-class laser pulses was a daunting prospect, but nevertheless one that was tackled in the US at the National Ignition Facility (NIF) ${ }^{[5]}$ and in France at Laser Mega-Joule (LMJ) ${ }^{[6]}$.

In the UK a novel approach was undertaken to break the problem into two parts; it was realized that, if it is possible to use one set of laser beams to compress a target and then a separate, much shorter, laser pulse at higher power to heat, then extreme conditions might be accessed with a much smaller laser than NIF or LMJ. Furthermore, such a laser system would be an ideal staging platform to larger lasers for collaboratively carrying out those experiments that do require megajoule class energies. This concept for the Orion laser facility combining short and long pulse lasers ${ }^{[7]}$ was endorsed, and building work commenced in 2006. 


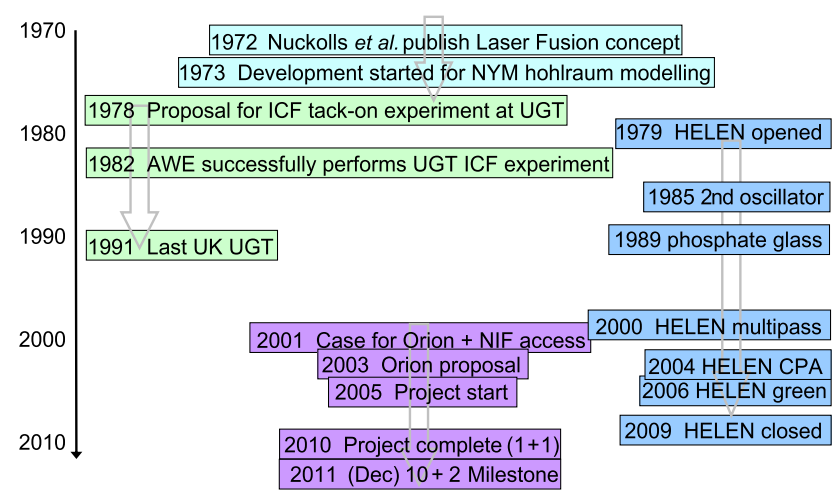

Figure 5. HEDP experiments at AWE followed dual tracks (albeit with a very limited number of experiments in the green underground testing (UGT) line) until nuclear testing stopped. HELEN had several upgrades, but has now been replaced by the Orion facility (NYM is a 2D hydrocode used for modelling and ICF is inertial confinement fusion).

Meanwhile, the HELEN laser undertook a final upgrade to prototype a chirped pulse amplification $(\mathrm{CPA})^{[8]}$ short pulse laser system. This system, similar to that demonstrated at the STFC Rutherford Appleton Laboratory's Central Laser Facility (CLF) Vulcan petawatt laser ${ }^{[9]}$, would be required for deployment on Orion. Experiments were very successful, and ultimately HELEN demonstrated opacity data at $500 \mathrm{eV}$ (around five million kelvin) ${ }^{[10]}$ for material at solid density. HELEN was closed in 2009 as it came time for Orion to enter its final commissioning phase. The high energy density physics timeline at AWE is illustrated schematically in Figure 5.

\section{The Orion laser/plasma interaction facility}

Orion effectively consists of two neodymium-doped glass laser systems. The 'long pulse' system (see Figure 6) comprises ten beamlines, each delivering a nominal $500 \mathrm{~J}$ of energy in a nanosecond with highly configurable pulse shape and relative timing. The beams are $300 \mathrm{~mm}$ in diameter and are generated and amplified at $1053 \mathrm{~nm}$ in the infrared before being frequency tripled to $351 \mathrm{~nm}$ (ultraviolet light) and then delivered to the target. The light enters the $4 \mathrm{~m}$ diameter target chamber in two cones of five beams at an angle of $50^{\circ}$ to the axis, allowing targets to be driven from either or both sides.

The 'short pulse' laser system consists of two beams each with an output beam diameter of $600 \mathrm{~mm}$. The beams start out at as low energy sub-picosecond pulses and are temporally stretched and amplified using the CPA technique which makes use of the small amount of spectral bandwidth available in the pulse. The stretched pulses can then undergo recompression using a pair of large diffraction gratings (940 $\mathrm{mm}$ diameter) and are delivered to the target at petawatt powers (500 $\mathrm{J}$ in $0.5 \mathrm{ps}$ ) and up to $10^{21} \mathrm{~W} \mathrm{~cm}^{-2}$ focused intensity.

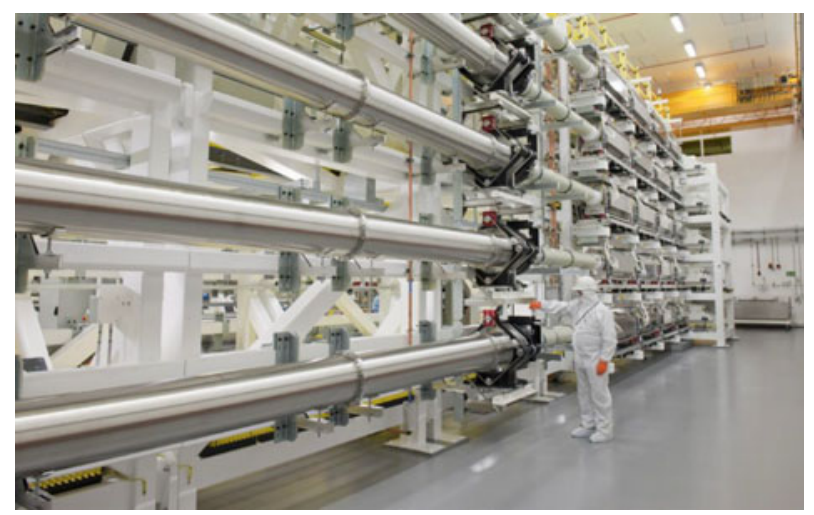

Figure 6. Orion's long pulse amplifier chain.

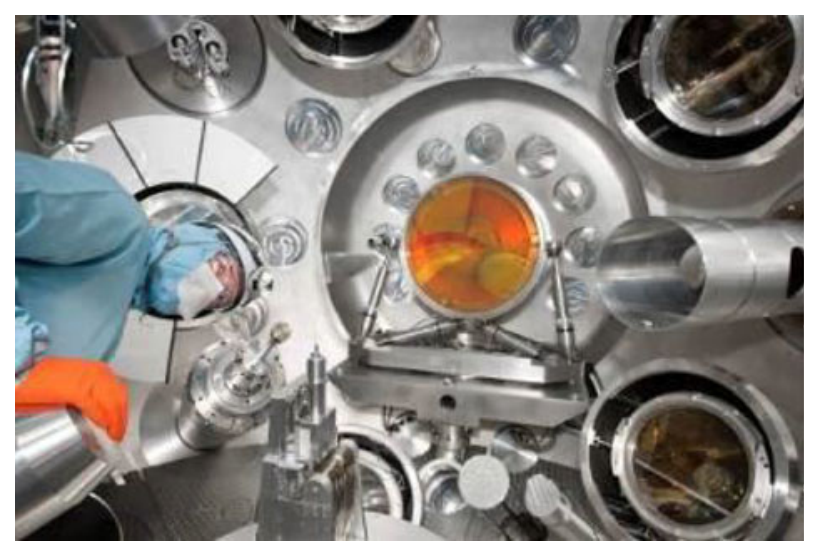

Figure 7. Inside the Orion target chamber.

The target chamber is shown in Figure 7, to which the two CPA beams are delivered orthogonally and can be used for heating or diagnostic purposes (a short pulse focusing parabola can be seen at the centre of Figure 7, coloured orange). One of the short pulse beamlines has the option of being converted to the second harmonic $(527 \mathrm{~nm})$ at a $300 \mathrm{~mm}$ sub-aperture, and thereby provides up to $100 \mathrm{~J}$ with a significantly reduced pre-pulse (i.e. higher contrast $\left.\sim 10^{-14}\right)^{[11]}$. A further upgrade providing a high contrast front-end to Orion has also recently been commissioned, providing unimaginably high contrast levels $\sim 10^{-18}$ when used in combination with the second harmonic option ${ }^{[12]}$.

In order to carry out experiments, the laser needs to be provided with a series of targets on which to focus its energy. These targets may be relatively simple flat layered structures or complex 3D assemblies depending on the experiment in question, see Figure 8. They are always very small, with typical dimensions from millimetres to microns, and with very close tolerance, typically microns to nanometres, and require the integration of material science, precision engineering, assembly, characterization and metrology for successful delivery. Some campaigns will require large numbers of these targets. 

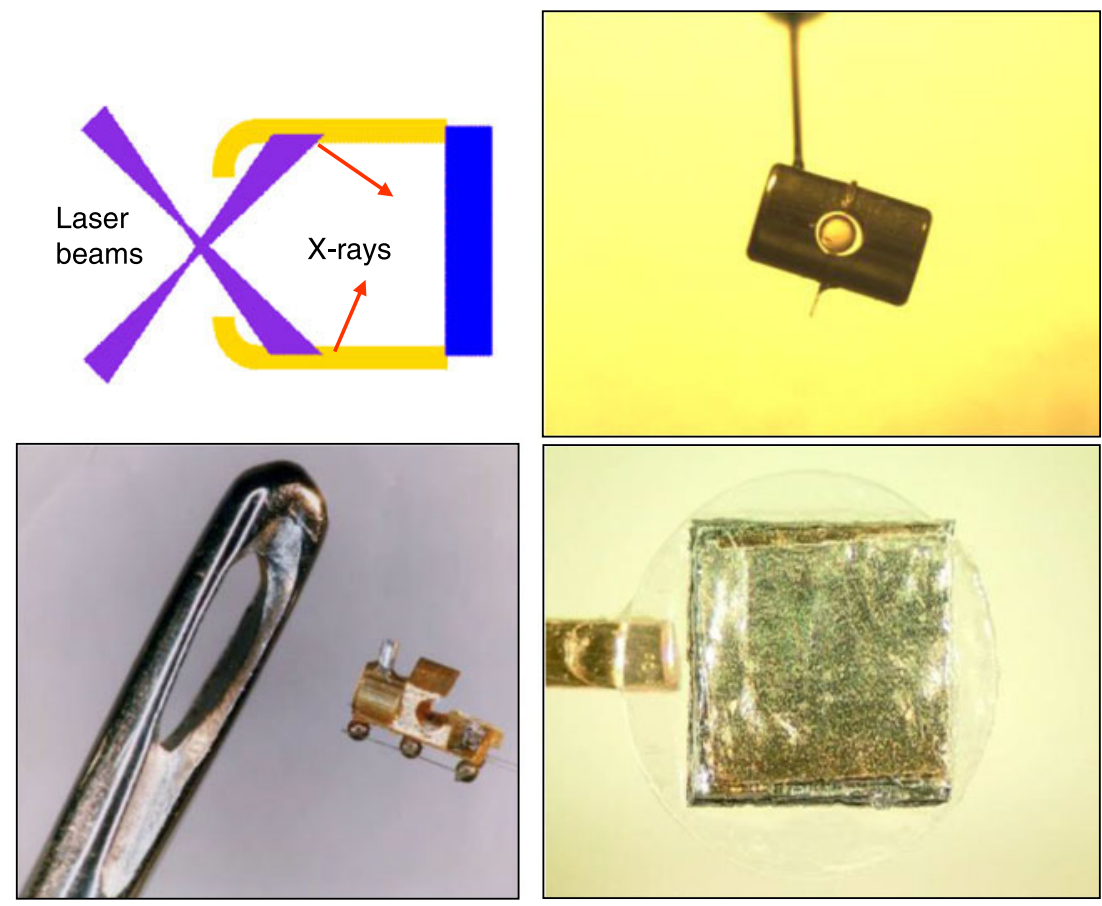

Figure 8. Targets range from 3D assemblies such as the hohlraum shown as a cartoon (top left) and as an actual target (top right) to flat foils (bottom right). They range in size, but the tolerances are always very tight. The image at the bottom left is a demonstration of target manufacturing capability on a miniature scale.

A laser facility also requires a range of diagnostics to provide useful data on the high energy density plasmas generated by the system. In principle, there is interest in measuring both the flux and the spectrum of any electromagnetic (optical, x-ray, etc.) or particle (electron, neutron, etc.) output from the target. The requirements, in each case, may be for time integrated or temporally resolved data, and spatial (or directional) resolution may also be required. The resolutions vary from experiment to experiment, but time scales as short as picoseconds and length scales of microns are not uncommon. For this reason, the Orion target chamber is equipped with many diagnostic ports, see Figure 9; some of these are committed to permanent diagnostics, but great flexibility is also provided by the use of six ten-inch manipulators (TIMs). Experimental teams can have access to instruments housed therein without needing to break the vacuum on the target chamber, and their use permits varied combinations of diagnostics to be fielded in different relative configurations.

The optical diagnostics on Orion include streak cameras, active and passive shock breakout systems, pyrometry and Velocity Interferometer System for Any Reflector (VISAR). The particle systems include electron spectrometers, Thomson parabolas, Faraday cups and Neutron Time of Flight (nToF) systems. There are also x-ray microscopes, a Dante soft x-ray system, a filter fluorescer (FFLEX), a transmission grating spectrometer, a hard $\mathrm{x}$-ray spectrometer and thermoluminescent dosimetry.

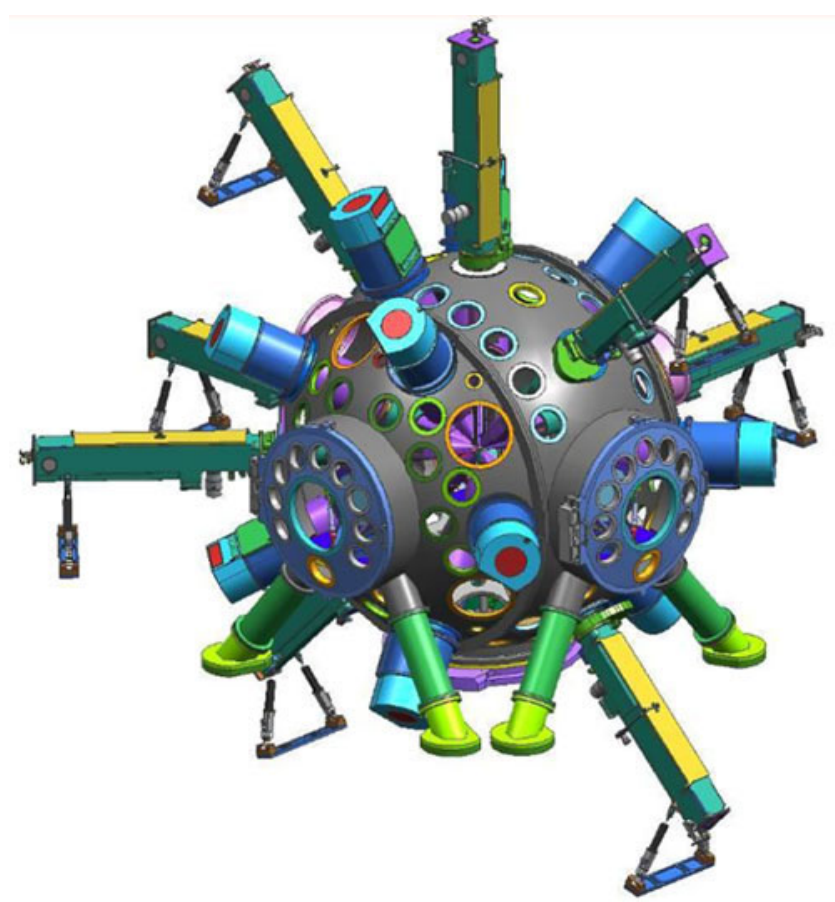

Figure 9. The Orion target chamber is peppered with ports, the majority being for diagnostic access. The TIMs are shown as the large oblong devices protruding from the chamber and the target inserter is mounted vertically at the top of the chamber.

The Orion laser was handed over from construction in December 2010 to enter a two-year commissioning programme. At the time of handover a mid-energy 
synchronization demonstration of one long pulse and one short pulse beam to the target had been carried out. By the end of the first year, the facility demonstrated all twelve beamlines delivering synchronized energy to the target chamber, and during the final commissioning period carried out the first opacity experiments on the facility as well as commissioning many auxiliary systems such as diagnostics.

The aim of the opacity experiments was to extend the experiments on HELEN, as described earlier, where aluminium was heated to $500 \mathrm{eV}$; by use of the combination of short and long pulse systems together, Orion is able to exceed these conditions. The experiment consisted of an aluminium foil sandwiched between two layers of plastic. When this foil is irradiated by the short pulse laser it is heated rapidly, and conditions are then diagnosed by x-ray spectroscopy. With much increased power and excellent contrast in the green, the Orion short pulse beam allows higher temperatures to be attained than on HELEN, but in particular it is possible to use the high energy long pulse laser beams to generate compression by launching a shock timed such that, after short pulse heating, the aluminium is at increased density as well as temperature ${ }^{[13,14]}$.

\section{Summary}

While we have emphasized the importance of Orion in certification under the CTBT, it will also be a very powerful and important tool to the wider UK scientific community and their international collaborators. It has already been stated that the physics of material properties in extreme conditions is an area of active interest in order to improve our understanding of stellar and planetary interiors. Similar conditions also exist in the inertial confinement fusion (ICF) capsules that could one day lead us to a world of clean energy, and research on Orion will be a useful staging platform for experiments in this context. To this end the facility is available for access by the scientific community for up to $15 \%$ of the available time, with access being prioritized through the Central Laser Facility's established processes.

At the point of writing, Orion had completed its first 18 months of operation, delivering a highly successful internal programme and having completed two academic experiments $^{[15]}$. These two campaigns were both from the University of Oxford;the first was an experiment to investigate materials under multi-megabar pressures led by Dr. Andrew Higginbotham and the second was to study the generation of plasma shock waves relevant to the conditions found in binary stars led by Prof. Gianluca Gregori. Preliminary analysis of the data obtained for both of these campaigns is very promising. Two further Orion academic access experiments have also been scheduled on Orion during 2014/2015: the investigation of colliding shocks relevant to astrophysics led by Dr. Francisco SuzukiVidal, Imperial College London, and a study of the effects of strong magnetic fields in plasmas using proton radiography led by Prof. Nigel Woolsey, University of York.

\section{Acknowledgements}

The work referred to in this paper has been carried out by members of the Plasma Physics and Design Physics departments at AWE, and they are therefore credited with the achievements described. The former heads of Plasma Physics, Peter Roberts, Daryl Landeg and Steve Rose, were central to the delivery of the Orion project.

\section{References}

1. K. O’Nions, R. Pitman, and C. Marsh, Nature 415, 853 (2002).

2. P. D. Roberts, AIP Conf. Proc. 1438, 35 (2012).

3. J. Nuckolls, L. Wood, A. Thiessen, and G. Zimmerman, Nature 239, 139 (1972).

4. M. J. Norman, J. E. Andrew, T. H. Bett, R. K. Clifford, J. E. England, N. W. Hopps, K. W. Parker, K. Porter, and M. Stevenson, Appl. Opt. 41, 3497 (2002).

5. G. H. Miller, E. I. Moses, and C. R. Wuest, Opt. Eng. 43, 2841 (2004).

6. J. Ebrardt and J. M. Chaput, J. Phys. Conf. Ser. 244, 032017 (2010).

7. N. W. Hopps, C. Danson, S. Duffield, D. Egan, S. Elsmere, M. Girling, E. Harvey, D. Hillier, M. Norman, S. Parker, P. Treadwell, D. Winter, and T. Bett, Appl. Opt. 52, 3597 (2013).

8. D. Strickland and G. Mourou, Opt. Commun. 56, 219 (1985).

9. C. N. Danson, P. A. Brummitt, R. J. Clarke, J. L. Collier, B. Fell, A. J. Frackiewicz, S. Hancock, S. Hawkes, C. Hernandez-Gomez, P. Holligan, M. H. R. Hutchinson, A. Kidd, W. J. Lester, I. O. Musgrave, D. Neely, D. R. Neville, P. A. Norreys, D. A. Pepler, C. J. Reason, W. Shaikh, T. B. Winstone, R. W. W. Wyatt, and B. E. Wyborn, Nucl. Fusion 44, S239 (2004).

10. C. R. D. Brown, D. J. Hoarty, S. F. James, D. Swatton, S. J. Hughes, J. W. Morton, T. M. Guymer, M. P. Hill, D. A. Chapman, J. E. Andrew, A. J. Comley, R. Shepherd, J. Dunn, H. Chen, M. Schneider, G. Brown, P. Beiersdorfer, and J. Emig, Phys. Rev. Lett. 106, 185003 (2011).

11. D. I. Hillier, C. N. Danson, S. J. Duffield, D. A. Egan, S. P. Elsmere, M. T. Girling, E. J. Harvey, N. W. Hopps, M. J. Norman, S. J. F. Parker, P. A. Treadwell, D. N. Winter, and T. H. Bett, Appl. Opt. 52, 18 (2013).

12. D. I. Hillier, S. Elsmere, M. Girling, N. Hopps, D. Hussey, S. Parker, P. Treadwell, D. Winter, and T. Bett, Appl. Opt. 53 (2014).

13. D. J. Hoarty, P. Allan, S. F. James, C. R. D. Brown, L. Hobbs, M. P. Hill, J. W. O. Harris, J. Morton, M. G. Brookes, R. Shepherd, J. Dunn, H. Chen, E. Von Marley, P. Beiersdorfer, G. Brown, and J. Emig, Phys. Rev. Lett. 110, 265003 (2013).

14. D. J. Hoarty, P. Allan, S. F. James, C. R. D. Brown, L. M. R. Hobbs, M. P. Hill, J. W. O. Harris, J. Morton, M. G. Brookes, R. Shepherd, J. Dunn, H. Chen, E. Von Marley, P. Beiersdorfer, H. K. Chung, R. W. Lee, G. Brown, and J. Emig, High Energy Density Physics 9, 661 (2013).

15. C. N. Danson, in Proceedings of 33rd ECLIM MoIO2 (2014). 